\title{
Nuclear symmetry potential in the relativistic impulse approximation
}

\author{
Zeng-Hua Li, ${ }^{1}$ Lie-Wen Chen,,${ }^{1,2}$ Che Ming Ko, ${ }^{3}$ Bao-An Li, ${ }^{4}$ and Hong-Ru Ma ${ }^{1}$ \\ ${ }^{1}$ Institute of Theoretical Physics, Shanghai Jiao Tong University, Shanghai 200240, People's Republic of China \\ ${ }^{2}$ Center of Theoretical Nuclear Physics, National Laboratory of Heavy-Ion Accelerator, Lanzhou 730000, People's Republic of China \\ ${ }^{3}$ Cyclotron Institute and Physics Department, Texas A\&M University, College Station, Texas 77843-3366, USA \\ ${ }^{4}$ Department of Physics, Texas A\&M University-Commerce, Commerce, Texas 75429, USA
}

(Received 30 June 2006; published 26 October 2006)

\begin{abstract}
Using the relativistic impulse approximation with the Love-Franey $N N$ scattering amplitude developed by Murdock and Horowitz, we investigate the low-energy $\left(100 \leqslant E_{\text {kin }} \leqslant 400 \mathrm{MeV}\right)$ behavior of the nucleon Dirac optical potential, the Schrödinger-equivalent potential, and the nuclear symmetry potential in isospin asymmetric nuclear matter. We find that the nuclear symmetry potential at fixed baryon density decreases with increasing nucleon energy. In particular, the nuclear symmetry potential at saturation density changes from positive to negative values at nucleon kinetic energy of about $200 \mathrm{MeV}$. Furthermore, the obtained energy and density dependence of the nuclear symmetry potential is consistent with those of the isospin- and momentum-dependent MDI interaction with $x=0$, which has been found to describe reasonably well both the isospin diffusion data from heavy-ion collisions and the empirical neutron-skin thickness of ${ }^{208} \mathrm{~Pb}$.
\end{abstract}

DOI: 10.1103/PhysRevC.74.044613

PACS number(s): 21.65.+f, 21.30.Fe, 24.10.Jv

\section{INTRODUCTION}

The energy dependence of the nuclear symmetry potential, i.e., the isovector part of nucleon mean-field potential in asymmetric nuclear matter, has recently attracted much attention [1-19]. Its knowledge together with that of the density dependence of the nuclear symmetry energy are important for understanding not only the structure of radioactive nuclei and the reaction dynamics induced by rare isotopes but also many critical issues in astrophysics [20,21]. Various microscopic and phenomenological models, such as the relativistic Dirac-Brueckner-Hartree-Fock（DBHF) [2,8,9,14,16,17,19] and the non-relativistic Brueckner-Hartree-Fock (BHF) [1,15] approach, the relativistic mean-field theory based on nucleonmeson interactions [13], and the nonrelativistic mean-field theory based on Skyrme-like interactions [3,12], have been used to study the nuclear symmetry potential, but the predicted results have varied widely. While most models predict a decreasing nuclear symmetry potential with increasing nucleon momentum, albeit at different rates, a few nuclear effective interactions used in some of the models lead to an opposite conclusion.

Using the relativistic Dirac optical potential obtained from the relativistic impulse approximation (RIA) [22-33] with the empirical nucleon-nucleon $(N N)$ amplitude calculated by McNeil, Ray, and Wallace (MRW) [22-24], which works well for elastic nucleon-nucleus scattering at medium and high energies (above $500 \mathrm{MeV}$ ), three of the present authors [34] have recently studied the high-energy behavior of the nuclear symmetry potential in asymmetric nuclear matter. It was found that for nucleons at high energies, the symmetry potential at fixed baryon density is essentially constant and slightly negative below nuclear densities of about $\rho=0.22 \mathrm{fm}^{-3}$, but it increases almost linearly to positive values at high densities.

A nice feature of RIA is that it permits very little phenomenological freedom in deriving the nucleon Dirac optical potential in nuclear matter. The basic ingredients in this method are the free $N N$ invariant scattering amplitude and the nuclear scalar and vector densities in nuclear matter. This is in contrast to the relativistic DBHF approach, where different approximation schemes and methods have been introduced for determining the Lorentz and isovector structure of the nucleon self-energy $[2,8,9,14,16,17,19]$. However, the original RIA of MRW failed to describe spin observables at laboratory energies lower than $500 \mathrm{MeV}$ [35], and its predicted oscillations in the analyzing power in proton- $\mathrm{Pb}$ scattering at large angles were also in sharp disagreement with experimental data [36]. These shortcomings are largely due to the implicit dynamical assumptions about the relativistic $N N$ interaction in the form of the Lorentz covariance [37] and the somewhat awkward behavior under the interchange of two particles [28] as well as the omitted medium modification due to the Pauli blocking effect. To solve these theoretical limitations at lower energies, Murdock and Horowitz (MH) [28,29] extended the original RIA to take into account the following three improvements: (i) an explicit exchange contribution was introduced by fitting to the relativistic $N N$ scattering amplitude, (ii) a pseudovector coupling rather than a pseudoscalar coupling was used for the pion, and (iii) a medium modification from the Pauli blocking was included. With these improvements, the RIA with free $N N$ scattering amplitude was then able to successfully reproduce the measured analyzing power and spin rotation function for all considered closed-shell nuclei in proton scattering near $200 \mathrm{MeV}$. Particularly, the medium modification due to the Pauli blocking effect was found to be essential in describing the spin rotation function for ${ }^{208} \mathrm{~Pb}$ at the proton energy of $290 \mathrm{MeV}$ [29].

Extending our previous work by using the generalized RIA of $\mathrm{MH}$ and the nuclear scalar and vector densities from the relativistic mean-field theory, we study in the present paper the low-energy $\left(100 \leqslant E_{\mathrm{kin}} \leqslant 400 \mathrm{MeV}\right)$ behavior of the nucleon Dirac optical potential, the Schrödinger-equivalent potential, and the nuclear symmetry potential in isospin asymmetric 
nuclear matter. We find that for low-energy nucleons, the nuclear symmetry potential at fixed nuclear density decreases with increasing nucleon energy. In particular, the nuclear symmetry potential at saturation density changes from positive to negative values at a nucleon kinetic energy of around $200 \mathrm{MeV}$. The resulting energy and density dependence of the nuclear symmetry potential is further found to be consistent with the isospin- and momentum-dependent MDI interaction with $x=0[3,10]$, which has been constrained by the isospin diffusion data in heavy-ion collisions and the empirical neutron-skin thickness of ${ }^{208} \mathrm{~Pb}[10,11,38,39]$. Our results thus provide an important consistency check for the energy dependence of the nuclear symmetry potential in asymmetric nuclear matter.

The paper is organized as follows. In Sec. II, we briefly review the generalized relativistic impulse approximation for the nuclear optical potential. Results on the relativistic nuclear optical potential, the resulting Schrödinger-equivalent potential, and the nuclear symmetry potential in asymmetric nuclear matter are presented in Sec. III. A summary is given in Sec. IV.

\section{THE THEORETICAL METHOD}

\section{A. The relativistic impulse approximation to the Dirac optical potential}

In the RIA, the momentum-space optical potential in a spin saturated nucleus is given by

$$
\tilde{U}(\mathbf{q})=\frac{-4 \pi i p_{\mathrm{lab}}}{M}\left[F_{S}(q) \tilde{\rho}_{S}(\mathbf{q})+\gamma_{0} F_{V}(q) \tilde{\rho}_{V}(\mathbf{q})\right],
$$

where $F_{S}$ and $F_{V}$ are, respectively, the scalar and the zeroth component of the vector $N N$ scattering amplitude; $\tilde{\rho}_{S}(\mathbf{q})$ and $\tilde{\rho}_{V}(\mathbf{q})$ are corresponding momentum-space nuclear densities; $p_{\text {lab }}$ and $M$ are, respectively, the laboratory momentum and mass of an incident nucleon; $\gamma_{0}$ is a Dirac $\gamma$ matrix; and $\mathbf{q}$ is the momentum transfer. The optical potential in the coordinator space is given by the Fourier transformation of $\tilde{U}(\mathbf{q})$. In infinite nuclear matter, the nuclear coordinate-space Dirac optical potential takes the simple form $[23,34]$

$$
U=\frac{-4 \pi i p_{\text {lab }}}{M}\left[F_{S 0} \rho_{S}+\gamma_{0} F_{V 0} \rho_{V}\right],
$$

where $F_{S 0} \equiv F_{S}(q=0)$ and $F_{V 0} \equiv F_{V}(q=0)$ are the $N N$ forward scattering amplitudes, while $\rho_{S}$ and $\rho_{V}$ are, respectively, the spatial scalar and vector densities of an infinite nuclear matter.

The Dirac optical potential in Eq. (2) is valid for nucleons at high energies. With decreasing nucleon energy, medium modification due to the Pauli blocking effect becomes important. As described in detail in Ref. [29], the Dirac optical potential including the Pauli blocking effect can be written as

$$
U_{\mathrm{opt}}=\left[1-a_{i}\left(E_{\mathrm{kin}}\right)\left(\frac{\rho_{B}}{\rho_{0}}\right)^{2 / 3}\right] U,
$$

where $\rho_{B}$ is the nuclear baryon density and $\rho_{0}=0.1934 \mathrm{fm}^{-3}$. The parameters $a_{i}\left(E_{\text {kin }}\right)$ denote the Pauli blocking factors for each energy $E_{\text {kin }}$ and are given in Table II of Ref. [29]. Although there are still many open questions on the role of medium modification [29], the $\rho_{B}^{2 / 3}$ density dependence of the Pauli blocking factor is consistent with the phasespace consideration for isotropic scattering [40]. For nucleon scattering in isospin asymmetric nuclear matter, the Pauli blocking effect becomes different for protons and neutrons. Following Ref. [40], we introduce an isospin-dependent Pauli blocking factor and obtain the following different Dirac optical potentials for protons and neutrons:

$U_{\mathrm{opt}}^{n(p)}=\left\{1-a_{i}\left(E_{\mathrm{kin}}\right)\left[\frac{\left(2 \rho_{n(p)}\right)^{2 / 3}+0.4\left(2 \rho_{p(n)}\right)^{2 / 3}}{1.4 \rho_{0}^{2 / 3}}\right]\right\} U^{n(p)}$.

Obviously, Eq. (4) reduces to Eq. (3) in symmetric nuclear matter with $\rho_{n}=\rho_{p}$.

\section{B. Nuclear scalar densities}

To evaluate the Dirac optical potential for nucleons in RIA, we need to know the nuclear scalar and vector densities. They can be determined from the relativistic mean-field (RMF) model [41]. Currently, there are many different versions for the RMF model, including mainly the nonlinear models [41-43], models with density-dependent meson-nucleon couplings [44-47], and point-coupling models [48-52]. As in Ref. [34], we use in the present work the nonlinear RMF model with a Lagrangian density that includes the nucleon field $\psi$, the isoscalar-scalar meson field $\sigma$, the isoscalar-vector meson field $\omega$, the isovector-vector meson field $\rho$, and the isovector-scalar meson field $\delta$, i.e.,

$$
\begin{aligned}
\mathcal{L}(\psi, \sigma, \omega, \rho, \delta)= & \bar{\psi}\left[\gamma_{\mu}\left(i \partial^{\mu}-g_{\omega} \omega^{\mu}\right)-\left(M-g_{\sigma} \sigma\right)\right] \psi \\
& +\frac{1}{2}\left(\partial_{\mu} \sigma \partial^{\mu} \sigma-m_{\sigma}^{2} \sigma^{2}\right)-\frac{1}{4} \omega_{\mu \nu} \omega^{\mu \nu} \\
& +\frac{1}{2} m_{\omega}^{2} \omega_{\mu} \omega^{\mu}-\frac{1}{3} b_{\sigma} M\left(g_{\sigma} \sigma\right)^{3} \\
& -\frac{1}{4} c_{\sigma}\left(g_{\sigma} \sigma\right)^{4}+\frac{1}{4} c_{\omega}\left(g_{\omega}^{2} \omega_{\mu} \omega^{\mu}\right)^{2} \\
& +\frac{1}{2}\left(\partial_{\mu} \delta \partial^{\mu} \delta-m_{\delta}^{2} \delta^{2}\right)+\frac{1}{2} m_{\rho}^{2} \rho_{\mu} \cdot \rho^{\mu} \\
& -\frac{1}{4} \rho_{\mu \nu} \cdot \rho^{\mu \nu}+\frac{1}{2}\left(g_{\rho}^{2} \rho_{\mu} \cdot \rho^{\mu}\right) \\
& \times\left(\Lambda_{S} g_{\sigma}^{2} \sigma^{2}+\Lambda_{V} g_{\omega}^{2} \omega_{\mu} \omega^{\mu}\right) \\
& -g_{\rho} \rho_{\mu} \bar{\psi} \gamma^{\mu} \tau \psi+g_{\delta} \delta \bar{\psi} \tau \psi,
\end{aligned}
$$

where the antisymmetric field tensors $\omega_{\mu \nu}$ and $\rho_{\mu \nu}$ are given by $\omega_{\mu \nu} \equiv \partial_{\nu} \omega_{\mu}-\partial_{\mu} \omega_{\nu}$ and $\rho_{\mu \nu} \equiv \partial_{\nu} \rho_{\mu}-\partial_{\mu} \rho_{\nu}$, respectively, and the symbols used in Eq. (5) have their usual meanings. The above Lagrangian density is quite general in the nonlinear RMF model and allows us to use many presently popular parameter sets.

In Ref. [34], three typical parameter sets were used to evaluate the scalar densities of neutrons and protons in asymmetric nuclear matter, and they are the very successful NL3 model [53], the Z271v model, and the HA model. The Z271v model has been used to study the neutron skin of heavy nuclei and the properties of neutron stars [54], while the HA model includes the isovector-scalar meson field $\delta$ and fits successfully some results calculated with the more microscopic DBHF approach [55]. As shown in Ref. [34], the scalar densities of neutrons and protons in asymmetric nuclear 
matter obtained from these three parameter sets are similar at low baryon densities but become different for $\rho_{B} \gtrsim 0.25 \mathrm{fm}^{-3}$, with Z271v giving a larger and NL3 a smaller scalar density than that from the parameter set HA. For $\rho_{B} \lesssim 0.25 \mathrm{fm}^{-3}$, the proton and neutron scalar densities from these three RMF models are also consistent with those from the RMF model with density-dependent meson-nucleon couplings [47]. The real and imaginary parts of the scalar potential at higher densities $\left(\rho_{B} \gtrsim 0.25 \mathrm{fm}^{-3}\right)$ thus depend on the interactions used in evaluating the nuclear scalar density and have, therefore, large uncertainties. In the present work, we only use the HA parameter set and focus on nuclear densities smaller than $\rho_{B} \lesssim 0.25 \mathrm{fm}^{-3}$ where the scalar densities of neutrons and protons in asymmetric nuclear matter are essentially independent of the model parameters.

\section{Nuclear symmetry potential}

In the Dirac spinor space of the projectile nucleon, the optical potential $U_{\text {opt }}$ is a $4 \times 4$ matrix and can be expressed in terms of a scalar $U_{S}^{\text {tot }}$ and a vector $U_{0}^{\text {tot }}$ piece:

$$
U_{\text {opt }}=U_{S}^{\text {tot }}+\gamma_{0} U_{0}^{\text {tot }} .
$$

Expressing $U_{S}^{\text {tot }}$ and $U_{0}^{\text {tot }}$ in terms of their real and imaginary parts, i.e.,

$$
U_{S}^{\mathrm{tot}}=U_{S}+i W_{S}, \quad U_{0}^{\mathrm{tot}}=U_{0}+i W_{0},
$$

a Schrödinger-equivalent potential (SEP) can be obtained from the Dirac optical potential [56,57]:

$$
U_{\mathrm{SEP}}=U_{S}^{\mathrm{tot}}+U_{0}^{\mathrm{tot}}+\frac{1}{2 M}\left(U_{S}^{\mathrm{tot} 2}-U_{0}^{\mathrm{tot} 2}\right)+\frac{U_{0}^{\mathrm{tot}}}{M} E_{\mathrm{kin}} .
$$

We note that solving the Schrödinger equation with the SEP gives the same bound-state energy eigenvalues and elastic phase shifts as the solution of the upper component of the Dirac spinor in the Dirac equation using the corresponding Dirac optical potential.

The real part of SEP is then given by

$$
\begin{aligned}
\operatorname{Re}\left(U_{\mathrm{SEP}}\right)= & U_{S}+U_{0}+\frac{1}{2 M}\left[U_{S}^{2}-W_{S}^{2}-\left(U_{0}^{2}-W_{0}^{2}\right)\right] \\
& +\frac{U_{0}}{M} E_{\mathrm{kin}} .
\end{aligned}
$$

The above equation corresponds to the nuclear mean-field potential in nonrelativistic models [16,58] and allows us to obtain the following nuclear symmetry potential, i.e., the so-called Lane potential [59],

$$
U_{\mathrm{sym}}=\frac{\operatorname{Re}\left(U_{\mathrm{SEP}}\right)_{n}-\operatorname{Re}\left(U_{\mathrm{SEP}}\right)_{p}}{2 \alpha},
$$

where $\operatorname{Re}\left(U_{\mathrm{SEP}}\right)_{n}$ and $\operatorname{Re}\left(U_{\mathrm{SEP}}\right)_{p}$ are, respectively, the real part of SEP for neutrons and protons. The isospin asymmetry $\alpha$ is defined as $\alpha=\frac{\rho_{n}-\rho_{p}}{\rho_{n}+\rho_{p}}$ with $\rho_{n}$ and $\rho_{p}$ denoting the neutron and proton densities, respectively.

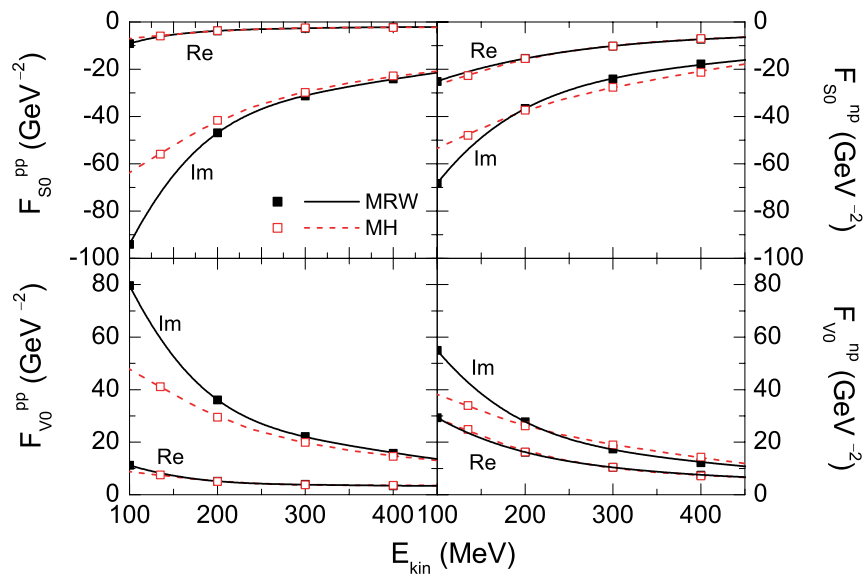

FIG. 1. (Color online) The scalar and vector parts of the free $N N$ forward scattering amplitudes $F_{S 0}^{p p}, F_{S 0}^{n p}, F_{V 0}^{p p}$, and $F_{V 0}^{n p}$ at nucleon kinetic energies $E_{\text {kin }}=135,200,300$, and $400 \mathrm{MeV}$ (open squares) from the RIA of MH. Dashed lines show polynomial fits to the energy dependence of the $N N$ scattering amplitude. Corresponding results from the original RIA of MRW are shown by solid squares and lines.

\section{RESULTS}

\section{A. Relativistic $N N$ scattering amplitude}

Based on the generalized RIA of MH with the Love-Franey $N N$ scattering amplitudes [60], we have evaluated the scalar and vector parts of the $N N$ forward scattering amplitudes $F_{S 0}^{p p}, F_{S 0}^{n p}, F_{V 0}^{p p}$, and $F_{V 0}^{n p}$ at nucleon kinetic energies $E_{\mathrm{kin}}=$ 135, 200, 300, and $400 \mathrm{MeV}$ for which the parameters can be found explicitly in Refs. [28,29]; the resulting values are shown by open squares in Fig. 1. To obtain continuous and smooth results for the $N N$ scattering amplitude and other quantities in the following, we made polynomial fits to the energy dependence of the $N N$ scattering amplitude, and the results are shown by dashed lines in Fig. 1. For comparison, we also include corresponding results from the original RIA of MRW. It is seen that for both proton-proton and proton-neutron scattering, the real parts of corresponding amplitudes in the two approaches are in good agreement with each other. However, for the imaginary parts of the amplitudes, the strength of the scalar and vector amplitudes from the RIA of MH displays much weaker energy dependence for both proton-proton and proton-neutron scattering at the energies $E_{\text {kin }} \leqslant 300 \mathrm{MeV}$. Since the imaginary part of the amplitude just corresponds to the real part of the Dirac optical potential as shown in Eq. (2), the above differences between the original RIA of MRW and the generalized RIA of MH thus lead to different behavior of the Dirac optical potential at low energies.

\section{B. Relativistic Dirac optical potential}

With free $N N$ forward scattering amplitudes of $\mathrm{MH}$ and MRW as well as the neutron and proton scalar and vector densities obtained from the RMF theory with the parameter set HA, we have investigated the real and imaginary parts of the scalar and vector Dirac optical potentials for nucleons in symmetric nuclear matter as functions of nucleon energy. In 


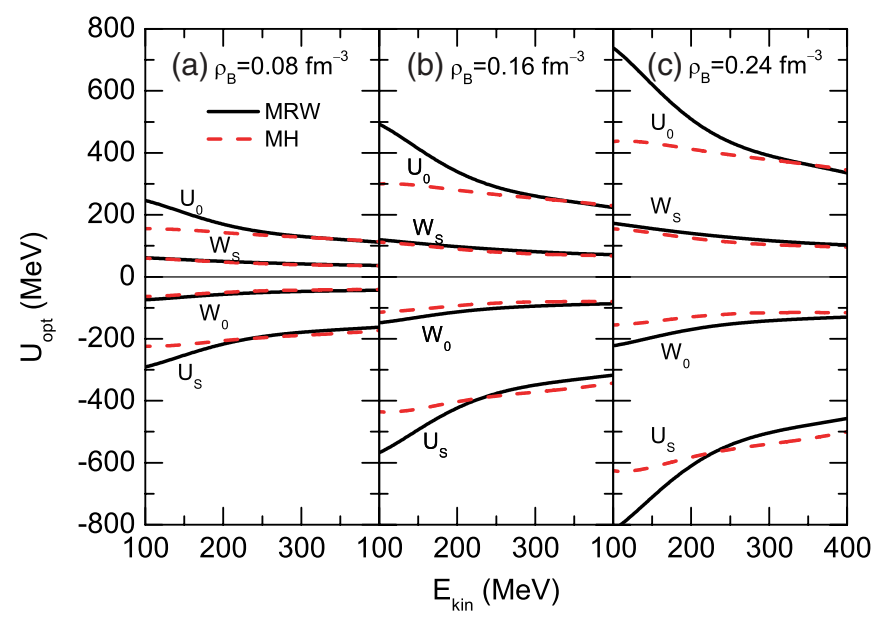

FIG. 2. (Color online) Energy dependence of the real and imaginary parts of the scalar and vector optical potentials in symmetric nuclear matter for different baryon densities $\rho_{B}$, with MH and MRW scattering amplitudes.

Fig. 2, the energy dependence of the Dirac optical potential is depicted at three nucleon densities $\rho_{\mathrm{B}}=0.08,0.16$, and $0.24 \mathrm{fm}^{-3}$. In each panel, we give the scalar and vector optical potentials based on the generalized amplitudes of $\mathrm{MH}$ and the original amplitudes of MRW. In calculating the Dirac optical potential from the RIA of MH, we included the Pauli blocking effect as well as the modifications from using the pseudovector coupling for pion and the exchange term contribution. For all considered densities, the energy dependence of the scalar and vector optical potentials from the RIA of MH are significantly reduced compared with those from the original RIA of MRW, especially for the real part at low energies. Furthermore, the difference between the two becomes larger with increasing density. These results thus demonstrate clearly the importance of the medium modifications introduced in the RIA of MH for nucleons at low energies. We also note that for all three considered densities, the RIA of MH generates, on the other hand, a similar systematic difference or isospin splitting in the Dirac optical potentials for protons and neutrons in asymmetric nuclear matter as in the original RIA of MRW [34]. In particular, the neutron exhibits stronger real but weaker imaginary scalar and vector potentials in neutron-rich nuclear matter.

\section{Schrödinger-equivalent optical potential}

Before discussing the nuclear symmetry potential, we first show in Fig. 3 the real part of the nucleon Schrödingerequivalent potential in symmetric nuclear matter at normal density obtained from the above Dirac optical potential. Because of uncertainties in the medium modification due to the Pauli blocking effect at low energies, results both with and without Pauli blocking corrections based on the $\mathrm{MH}$ free $N N$ scattering amplitudes are shown. For comparison, we also show the real part of the nucleon Schrödinger-equivalent potential from the original RIA of MRW, which is seen to be always positive at the considered energy range of $E_{\text {kin }}=100 \sim 400 \mathrm{MeV}$. Including the pseudovector coupling

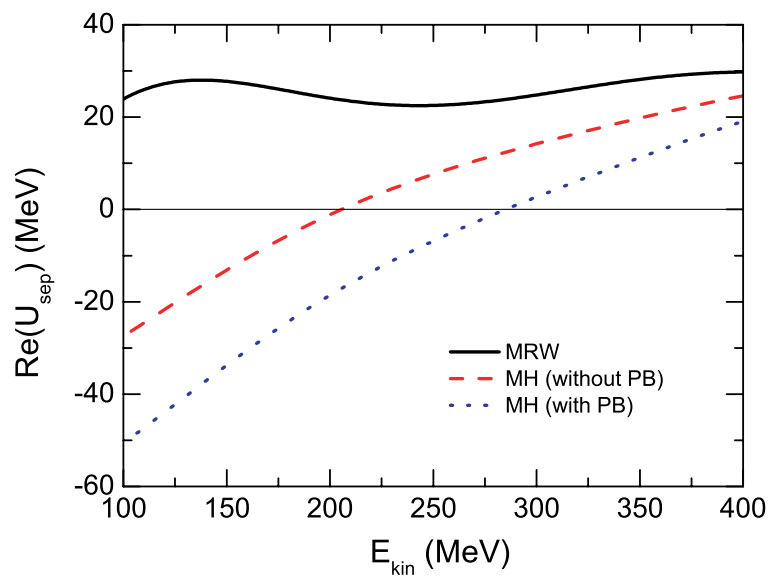

FIG. 3. (Color online) Energy dependence of the real part for the nucleon Schrödinger-equivalent potential at normal density in symmetric nuclear matter, from the original RIA of MRW and from the RIA of MH with and without Pauli blocking correction.

and exchange term corrections in the RIA of MH (dashed line), the behavior of the resulting Schrödinger-equivalent potential as a function of energy is significantly improved, varying from $-27 \mathrm{MeV}$ at $E_{\text {kin }}=100 \mathrm{MeV}$ to $0 \mathrm{MeV}$ at $E_{\text {kin }} \approx 200 \mathrm{MeV}$ and then continues to increase monotonously as the nucleon energy increases. This improvement is due to the fact that the pseudovector coupling and exchange term corrections lead to a smaller strength of the imaginary scalar and vector $N N$ forward scattering amplitudes while keeping their sum roughly unchanged as shown in Fig. 1. From Eq. (2), therefore, the term $U_{S}+U_{0}$ does not change while the last two terms in Eq. (9) are reduced strongly and thus a smaller Schrödinger-equivalent potential is obtained. When the Pauli blocking effect is further taken into account, the resulting Schrödinger-equivalent potential is seen to be more attractive at the whole energy range considered here. At high enough energy, the Schrödinger-equivalent potentials from the above three approaches become similar as expected since effects from both Pauli blocking and exchange contribution play minor roles at high energies.

We note that with momentum/energy-independent scalar and vector potentials from the RMF calculation, the nucleon Schrödinger-equivalent potential in symmetric nuclear matter at normal nuclear density already exhibits a linear energy dependence according to Eq. (9), with a change from negative to positive values typically at kinetic energies between about 200 and $500 \mathrm{MeV}$ depending on the model parameters [61]. Experimental data from the global relativistic optical-model analysis also indicate that the nucleon Schrödinger-equivalent potential in symmetric nuclear matter at normal nuclear density changes from negative to positive values around $200 \mathrm{MeV}$, although with large uncertainties, as mentioned in Ref. [61].

\section{Nuclear symmetry potential}

For the nuclear symmetry potential based on the scattering amplitudes of MH, we show in Fig. 4 its energy dependence for both cases of using isospin-dependent [Eq. (4)] and isospin-independent Pauli blocking [Eq. (3)] corrections at 


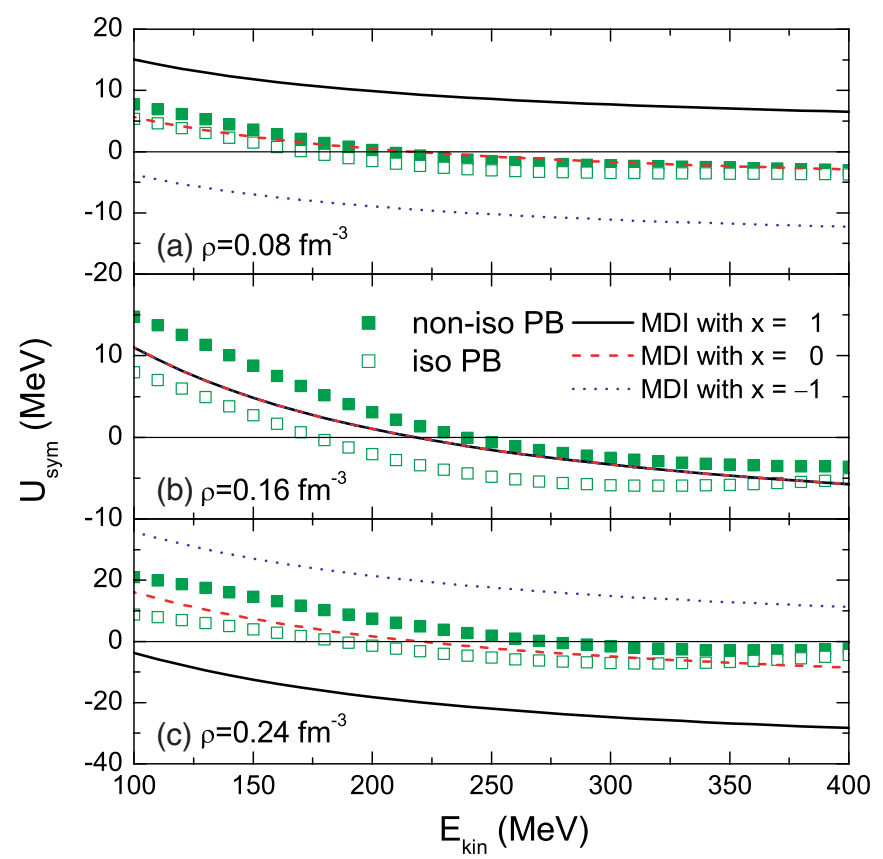

FIG. 4. (Color online) Energy dependence of the nuclear symmetry potential from the RIA of MH with isospin-dependent (open squares) and isospin-independent (solid squares) Pauli blocking corrections, as well as the phenomenological MDI interaction with $x=1,0$, and -1 at three fixed baryon densities.

fixed baryon densities of $\rho_{B}=0.08,0.16$, and $0.24 \mathrm{fm}^{-3}$. Also shown are results from the phenomenological parametrization of the isospin- and momentum-dependent nuclear mean-field potential, i.e., the MDI interaction with $x=-1,0$, and 1 , which has recently been extensively used in the transport model for studying isospin effects in intermediate-energy heavy-ion collisions induced by neutron-rich nuclei $[5,6,10,11$, 62-65]. In the MDI interaction, the single nucleon potential in asymmetric nuclear matter with isospin asymmetry $\alpha$ is expressed by $[3,5,6,10,11]$

$$
\begin{aligned}
U(\rho, \alpha, \mathbf{p}, \tau, \mathbf{r})= & \left(-95.98-x \frac{2 B}{\sigma+1}\right) \frac{\rho_{\tau^{\prime}}}{\rho_{0}} \\
& +\left(-120.57+x \frac{2 B}{\sigma+1}\right) \frac{\rho_{\tau}}{\rho_{0}} \\
& +B\left(\frac{\rho}{\rho_{0}}\right)^{\sigma}\left(1-x \alpha^{2}\right) \\
& -8 \tau x \frac{B}{\sigma+1} \frac{\rho^{\sigma-1}}{\rho_{0}^{\sigma}} \alpha \rho_{\tau^{\prime}}+\frac{2 C_{\tau, \tau}}{\rho_{0}} \\
& \times \int d^{3} \mathbf{p}^{\prime} \frac{f_{\tau}\left(\mathbf{r}, \mathbf{p}^{\prime}\right)}{1+\left(\mathbf{p}-\mathbf{p}^{\prime}\right)^{2} / \Lambda^{2}}+\frac{2 C_{\tau, \tau^{\prime}}}{\rho_{0}} \\
& \times \int d^{3} \mathbf{p}^{\prime} \frac{f_{\tau^{\prime}}\left(\mathbf{r}, \mathbf{p}^{\prime}\right)}{1+\left(\mathbf{p}-\mathbf{p}^{\prime}\right)^{2} / \Lambda^{2}} .
\end{aligned}
$$

In this equation, $\tau=1 / 2(-1 / 2)$ for neutrons (protons) and $\tau \neq \tau^{\prime} ; \sigma=4 / 3$; and $f_{\tau}(\mathbf{r}, \mathbf{p})$ is the phase-space distribution function at coordinate $\mathbf{r}$ and momentum $\mathbf{p}$. The parameters $B, C_{\tau, \tau}, C_{\tau, \tau^{\prime}}$, and $\Lambda$ are determined by fitting the momentum dependence of $U(\rho, \alpha, \mathbf{p}, \tau, \mathbf{r})$ to that predicted by the Gogny-Hartree-Fock and/or the Brueckner-HartreeFock (BHF) calculations [1], the saturation properties of symmetric nuclear matter, and the symmetry energy of $31.6 \mathrm{MeV}$ at normal nuclear matter density $\rho_{0}=0.16 \mathrm{fm}^{-3}$ [3]. The incompressibility $K_{0}$ of symmetric nuclear matter at $\rho_{0}$ is set to be $211 \mathrm{MeV}$. The different $x$ values in the MDI interaction are introduced to vary the density dependence of the nuclear symmetry energy while keeping other properties of the nuclear equation of state fixed [10]. We note that the energy dependence of the symmetry potential from the MDI interaction is consistent with the empirical Lane potential at normal nuclear matter density and low nucleon energies [4].

It is seen from Fig. 4 that at fixed baryon density, the nuclear symmetry potential generally decreases with increasing nucleon energy. At low nuclear density $\left(\rho_{\mathrm{B}}=0.08\right.$ $\mathrm{fm}^{-3}$ ), the symmetry potentials from the RIA of $\mathrm{MH}$ with isospin-dependent and isospin-independent Pauli blocking corrections are almost the same, especially at energies higher than $E_{\text {kin }} \geqslant 300 \mathrm{MeV}$, where the Pauli blocking correction is expected to be unimportant. The isospin dependence of the Pauli blocking effect, however, becomes stronger as nuclear density increases, and an appreciable difference in the resulting symmetry potentials is seen. The difference disappears, though, for high-energy nucleons when the Pauli blocking effect becomes negligible. It is interesting to note that at normal density $\left(\rho_{\mathrm{B}}=0.16 \mathrm{fm}^{-3}\right)$, the nuclear symmetry potential changes from positive to negative values at nucleon kinetic energy around $200 \mathrm{MeV}$, with the one using the isospin-dependent Pauli blocking correction at a somewhat lower energy than that using the isospin-independent Pauli blocking correction. Comparing with results from the MDI interaction, the one with $x=0$ is seen in surprisingly good agreement with the results of RIA by $\mathrm{MH}$ in the region of nuclear densities and energies considered here. Although the MDI interaction with different $x$ values give by construction the same symmetry potential at normal nuclear matter density as shown in Fig. 4(b), the one with $x=0$ has been found to give reasonable descriptions of the data on the isospin diffusion in intermediate energy heavy-ion collisions and the neutron-skin thickness of ${ }^{208} \mathrm{~Pb}[10,11,38,39]$.

The density dependence of the nuclear symmetry potential with isospin-dependent and isospin-independent Pauli blocking corrections at nucleon kinetic energies of 100, 200, 300, $400 \mathrm{MeV}$ are shown in Fig. 5 together with corresponding results from the MDI interaction with $x=-1,0$, and 1 . It is clearly seen that the nuclear symmetry potentials are always positive at lower nucleon kinetic energy of $E_{\text {kin }}=100 \mathrm{MeV}$ while it may become positive or negative at $E_{\mathrm{kin}}=200 \mathrm{MeV}$ depending on whether the Pauli blocking effect is isospin dependent or not. At higher energies $\left(E_{\text {kin }}=300\right.$ and $\left.400 \mathrm{MeV}\right)$, the nuclear symmetry potential is always negative in the density region considered here. These features are consistent with the results shown in Fig. 4. Compared with results from the MDI interaction, it is seen that the nuclear symmetry potential from the generalized RIA of $\mathrm{MH}$ reproduces nicely the results obtained from the MDI interaction with $x=0$ when $\rho_{\mathrm{B}} \lesssim 0.2 \mathrm{fm}^{-3}$ even for nucleon kinetic energy as high as $400 \mathrm{MeV}$. Moreover, in the energy region of $E_{\mathrm{kin}}=100 \sim 300 \mathrm{MeV}$, the nuclear 


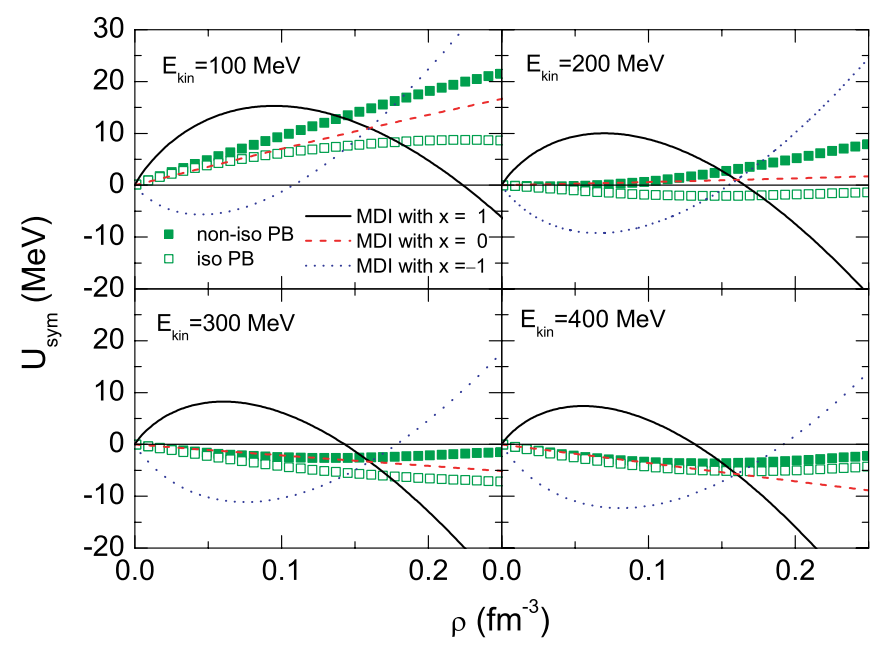

FIG. 5. (Color online) Density dependence of the nuclear symmetry potential using RIA with isospin-dependent and isospinindependent Pauli blocking, as well as the results from the phenomenological interaction MDI with $x=-1,0$, and 1 at four nucleon kinetic energies.

symmetry potential from MDI interaction with $x=0$ always lies between the results from the RIA of MH with isospindependent and isospin-independent Pauli blocking corrections. On the other hand, the MDI interaction with both $x=-1$ and 1 display very different density dependence from the results using the RIA of MH.

\section{SUMMARY}

Based on the generalized relativistic impulse approximation of $\mathrm{MH}$ and the scalar and vector densities from the relativistic mean-field theory with the parameter set HA, we have studied the low-energy behavior of the nuclear symmetry potential in asymmetric nuclear matter. In the relativistic impulse approximation of $\mathrm{MH}$, the low-energy behavior of the Dirac optical potential has been significantly improved by including the pseudovector coupling for pion, exchange contribution, and medium modification caused by the Pauli blocking effect. We find that compared with results from the original RIA of MRW, the generalized RIA of MH gives essentially identical real parts of the scalar and vector amplitudes for both proton-proton and neutron-proton scattering but significantly reduced strength in their imaginary parts at low energies $E_{\text {kin }} \leqslant 300 \mathrm{MeV}$. These improvements in the RIA of MH modify the real scalar and vector Dirac optical potentials at lower energies and make the resulting energy dependence of the Schrödinger-equivalent potential and nuclear symmetry potential more reasonable.
At saturation density, the nuclear symmetry potential is found to change from positive to negative values at a nucleon kinetic energy of about $200 \mathrm{MeV}$. This is a very interesting result as it implies that the proton (neutron) feels an attractive (repulsive) symmetry potential at lower energies but a repulsive (attractive) symmetry potential at higher energies in asymmetric nuclear matter. Adding also the repulsive Coulomb potential, a high-energy proton in asymmetric nuclear matter thus feels a very stronger repulsive potential. This behavior of the nuclear symmetry potential can be studied in intermediateand high-energy heavy-ion collisions that are induced by radioactive nuclei, e.g., by measuring two-nucleon correlation functions [66] and light cluster production [67] in these collisions.

Comparing the energy and density dependence of the nuclear symmetry potential from the RIA of $\mathrm{MH}$ with that from the MDI interaction indicates that results from the MDI interaction with $x=0$ are in good agreement with those from the RIA of MH. For baryon density less than $0.25 \mathrm{fm}^{-3}$ and nucleon energy less than $400 \mathrm{MeV}$ as considered in the present work, the nuclear symmetry potential from the MDI interaction with $x=0$ lies approximately between the two results from the RIA of MH with isospin-dependent and isospin-independent Pauli blocking corrections. This provides strong evidence for the validity of the MDI interaction with $x=0$ in describing both the isospin diffusion data in intermediate-energy heavyion collisions and the neutron-skin thickness data for ${ }^{208} \mathrm{~Pb}$.

The results presented in the present work thus provide an important consistency check for the energy/momentum dependence of the nuclear symmetry potential in asymmetric nuclear matter, particularly the MDI interaction with $x=0$, which is an essential input to the isospin-dependent transport model $[5,11,13]$ in studying heavy-ion collisions induced by radioactive nuclei at intermediate energies. They will also be useful in future studies that extend the Lorentz-covariant transport model $[68,69]$ to include explicitly the isospin degrees of freedom.

\section{ACKNOWLEDGMENTS}

This work was supported in part by the Post-doctors Research Award Fund of China, the National Natural Science Foundation of China under Grant Nos. 10334020, 10575071, and 10675082, MOE of China under project NCET-05-0392, Shanghai Rising-Star Program under Grant No. 06QA14024, the U.S. National Science Foundation under Grant Nos. PHY-0457265, PHY-0354572, and PHY-0456890, the Welch Foundation under Grant No. A-1358, and the NASA-Arkansas Space Grants Consortium Award ASU15154.
[1] I. Bombaci and U. Lombardo, Phys. Rev. C 44, 1892 (1991).

[2] S. Ulrych and H. Müther, Phys. Rev. C 56, 1788 (1997).

[3] C. B. Das, S. Das Gupta, C. Gale, and B. A. Li, Phys. Rev. C 67, 034611 (2003).

[4] B. A. Li, Phys. Rev. C 69, 064602 (2004).

[5] B. A. Li, C. B. Das, S. Das Gupta, and C. Gale, Phys. Rev. C 69, 011603(R) (2004); Nucl. Phys. A735, 563 (2004).
[6] L. W. Chen, C. M. Ko, and B. A. Li, Phys. Rev. C 69, 054606 (2004).

[7] J. Rizzo, M. Colonna, M. Di Toro, and V. Greco, Nucl. Phys. A732, 202 (2004).

[8] E. N. E. van Dalen, C. Fuchs, and A. Faessler, Nucl. Phys. A744, 227 (2004).

[9] Z. Y. Ma, J. Rong, B. Q. Chen, Z. Y. Zhu, and H. Q. Song, Phys. Lett. B604, 170 (2004). 
[10] L. W. Chen, C. M. Ko, and B. A. Li, Phys. Rev. Lett. 94, 032701 (2005).

[11] B. A. Li and L. W. Chen, Phys. Rev. C 72, 064611 (2005).

[12] B. Behera, T. R. Routray, A. Pradhan, S. K. Patra, and P. K. Sahu, Nucl. Phys. A753, 367 (2005).

[13] V. Baran, M. Colonna, V. Greco, and M. Di Toro, Phys. Rep. 410, 335 (2005).

[14] F. Sammarruca, W. Barredo, and P. Krastev, Phys. Rev. C 71, 064306 (2005).

[15] W. Zuo, L. G. Cao, B. A. Li, U. Lombardo, and C. W. Shen, Phys. Rev. C 72, 014005 (2005).

[16] E. N. E. van Dalen, C. Fuchs, and A. Faessler, Phys. Rev. Lett. 95, 022302 (2005).

[17] E. N. E. van Dalen, C. Fuchs, and A. Faessler, Phys. Rev. C 72, 065803 (2005).

[18] J. Rizzo, M. Colonna, and M. Di Toro, Phys. Rev. C 72, 064609 (2005).

[19] J. Rong, Z. Y. Ma, and N. Van Giai, Phys. Rev. C 73, 014614 (2006).

[20] B. A. Li, C. M. Ko, and W. Bauer, Int. J. Mod. Phys. E 7, 147 (1998).

[21] Isospin Physics in Heavy-Ion Collisions at Intermediate Energies, edited by Bao-An Li and W. Udo Schröder (Nova Science, New York, 2001).

[22] J. A. McNeil, L. Ray, and S. J. Wallace, Phys. Rev. C 27, 2123 (1983).

[23] J. A. McNeil, J. R. Shepard, and S. J. Wallace, Phys. Rev. Lett. 50, 1439 (1983).

[24] J. R. Shepard, J. A. McNeil, and S. J. Wallace, Phys. Rev. Lett. 50, 1443 (1983).

[25] L. G. Arnold, B. C. Clark, and R. L. Mercer, Phys. Rev. C 19, 917 (1979).

[26] B. C. Clark, S. Hama, R. L. Mercer, L. Ray, and B. D. Serot, Phys. Rev. Lett. 50, 1644 (1983).

[27] L. D. Miller, Phys. Rev. Lett. 51, 1733 (1983).

[28] C. J. Horowitz, Phys. Rev. C 31, 1340 (1985).

[29] D. P. Murdock and C. J. Horowitz, Phys. Rev. C 35, 1442 (1987).

[30] J. A. Tjon and S. J. Wallace, Phys. Rev. C 32, 267 (1985); 35, 280 (1987); 36, 1085 (1987).

[31] N. Ottenstein, S. J. Wallace, and J. A. Tjon, Phys. Rev. C 38, 2272 (1988).

[32] Y. Jin and R. W. Finlay, Phys. Rev. C 47, 1697 (1993).

[33] K. Kaki and H. Toki, Nucl. Phys. A696, 453 (2001); K. Kaki, H. Toki, and I. Tanihata, ibid. A724, 99 (2003).

[34] L. W. Chen, C. M. Ko, and B. A. Li, Phys. Rev. C 72, 064606 (2005).

[35] L. Ray and G. W. Hoffmann, Phys. Rev. C 31, 538 (1985).

[36] T. Drake, in Medium Energy Nucleon and Antinucleon Scattering, Bad Honnef, West Germany, 1985, edited by H. V. von Geramb (Springer-Verlag, Berlin, 1985).

[37] D. L. Adams and M. Bleszynski, Phys. Lett. B136, 10 (1984).

[38] A. W. Steiner and B. A. Li, Phys. Rev. C 72, 041601(R) (2005).
[39] L. W. Chen, C. M. Ko, and B. A. Li, Phys. Rev. C 72, 064309 (2005).

[40] L. W. Chen, F. S. Zhang, Z. H. Lu, and H. R. Ma, Phys. Rev. C 64, 064315 (2001).

[41] B. D. Serot and J. D. Walecka, Adv. Nucl. Phys. 16, 1 (1986); Int. J. Mod. Phys. E 6, 515 (1997).

[42] P.-G. Reinhard, Rep. Prog. Phys. 52, 439 (1989).

[43] P. Ring, Prog. Part. Nucl. Phys. 37, 193 (1996).

[44] C. Fuchs, H. Lenske, and H. H. Wolter, Phys. Rev. C 52, 3043 (1995).

[45] H. Shen, Y. Sugahara, and H. Toki, Phys. Rev. C 55, 1211 (1997).

[46] S. Typel and H. H. Wolter, Nucl. Phys. A656, 331 (1999).

[47] F. Hofmann, C. M. Keil, and H. Lenske, Phys. Rev. C 64, 034314 (2001).

[48] J. J. Rusnak and R. J. Furnstahl, Nucl. Phys. A627, 495 (1997).

[49] T. Bürvenich, D. G. Madland, J. A. Maruhn, and P.-G. Reinhard, Phys. Rev. C 65, 044308 (2002).

[50] D. G. Madland, T. Bürvenich, J. A. Maruhn, and P.-G. Reinhard, Nucl. Phys. A741, 52 (2004).

[51] T. Bürvenich, D. G. Madland, and P.-G. Reinhard, Nucl. Phys. A744, 92 (2004).

[52] P. Finelli, N. Kaiser, D. Vretenar, and W. Weise, Nucl. Phys. A735, 449 (2004).

[53] G. A. Lalazissis, J. König, and P. Ring, Phys. Rev. C 55, 540 (1997).

[54] C. J. Horowitz, and J. Piekarewicz, Phys. Rev. Lett. 86, 5647 (2001); Phys. Rev. C 64, 062802(R) (2001); 66, 055803 (2002).

[55] J. K. Bunta and S. Gmuca, Phys. Rev. C 68, 054318 (2003).

[56] R. Brockmann, Phys. Rev. C 18, 1510 (1978).

[57] M. Jaminon, C. Mahaux, and P. Rochus, Phys. Rev. C 22, 2027 (1980); Nucl. Phys. A365, 371 (1981).

[58] M. Jaminon and C. Mahaux, Phys. Rev. C 40, 354 (1989).

[59] A. M. Lane, Nucl. Phys. 35, 676 (1962).

[60] W. G. Love and M. A. Franey, Phys. Rev. C 24, 1073 (1981); 27, 438 (1983).

[61] K. Weber, B. Blättel, W. Cassing, H. C. Dönges, V. Koch, A. Lang, and U. Mosel, Nucl. Phys. A539, 713 (1992).

[62] B. A. Li, G. C. Yong, and W. Zuo, Phys. Rev. C 71, 014608 (2005); 71, 044604 (2005).

[63] B. A. Li, L. W. Chen, G. C. Yong, and W. Zuo, Phys. Lett. B634, 378 (2006).

[64] G. C. Yong, B. A. Li, L. W. Chen, and W. Zuo, Phys. Rev. C 73, 034603 (2006).

[65] G. C. Yong, B. A. Li, and L. W. Chen, Phys. Rev. C (2006), in press [arXiv:nucl-th/0606003].

[66] L. W. Chen, V. Greco, C. M. Ko, and B. A. Li, Phys. Rev. Lett. 90, 162701 (2003); Phys. Rev. C 68, 014605 (2003).

[67] L. W. Chen, C. M. Ko, and B. A. Li, Phys. Rev. C 68, 017601 (2003); Nucl. Phys. A729, 809 (2003).

[68] C. M. Ko, Q. Li, and R. Wang, Phys. Rev. Lett. 59, 1084 (1987); C. M. Ko and Q. Li, Phys. Rev. C 37, 2270 (1988); C. M. Ko and G. Q. Li, J. Phys. G 22, 1673 (1996).

[69] T. Maruyama, B. Blättel, W. Cassing, A. Lang, U. Mosel, and K. Weber, Phys. Lett. B297, 228 (1992). 